\title{
Relación entre los estadios de maduración esqueletal y calcificación dentaria
}

\section{Relationship between stages of skeletal maturation and tooth calcification}

\author{
Ríos Villasis LK*, Soldevilla Galarza L**
}

\section{RESUMEN}

Introducción: El propósito del estudio fue determinar el grado de correlación entre los estadios de calcificación dentaria según Demirjian para caninos, primeras y segundas premolares inferiores, y los estadios de maduración ósea de Fishman (curva de crecimiento puberal).

Material y métodos: La muestra consistió en 72 pares de radiografías panorámicas y carpales, 41 de hombres (56,94\%) y 31 de mujeres (43,06\%), tomadas el mismo día. La muestra se agrupó según sexo y lado a que pertenecía cada diente analizado. Se utilizaron la prueba de correlación de Spearman y tablas de distribución de frecuencia.

Resultados: Las correlaciones encontradas fueron significativas en todos los casos; la correlación más alta respecto a Fishman se dio con el canino izquierdo $(0,766)$, y la más baja con el segundo premolar izquierdo $(0,581)$, la más alta correlación en cuanto a calcificación dentaria se dio entre caninos de ambos lados $(0,985)$. El estadio G de calcificación dentaria según Demirjian, coincidió con el pico máximo de crecimiento puberal en mujeres (estadio 6 de Fishman); y en hombres coincidió con la calcificación del sesamoideo (estadio 4 de Fishman).

Conclusiones: Se concluye que existe correlación significativa entre los estadios de maduración ósea de Fishman y los estadios de calcificación dentaria.

Palabras clave: Calcificación del diente, crecimiento y desarrollo, diente canino, maduración ósea.

\section{SUMMARY}

Introduction: The purposeof the study was to determine the degree of correlation between the Demirjian Stages of Calcification of mandibular cuspids, first and second bicuspids and the stages of carpal bone maturation Fishman.

Material y methods: The sample calculated at a level of significance of $95 \%(p<0.05)$ consisted of double sets of panoramic and wrist radiographs (41 males, 56.94\% y 31 females $43.06 \%$ ) taken the same day. The sample was subdivided according to sex and the side to which each tooth analyzed belonged. Spearman correlation test and tables of frequency distribution were used.

Results: The correlation found was of significance in all cases, with the higher Fishman correlation found in the left cuspid (0.766) and the lowest correlation in the right second bicuspid (0.581). The higher correlation due to dental calcification was found between the cupids of both sides (0.985). Demirjian's G calcification stage coincided with the female pubertal growth spurt (stage 6 of Fishman), and males coincided with calcification the sesamoid (stage 4 of Fishman).

* Magíster en Estomatología. Universidad Peruana Cayetano Heredia. Cirujano dentista egresada de la Universidad Nacional Mayor de San Marcos. Docente de la Universidad Inca Garcilaso de la Vega.

** Especialista en Ortodoncia. Docente titular en Ortodoncia de la Facultad de Odontología de la Universidad Nacional Mayor de San Marcos. 
Conclusion: We conclude that there is a high significance correlation between the stages of calcification of mandibular cuspids, first and second bicuspids and the stages of carpal bone maturation Fishman.

Key words: Tooth calcification, growth and development, cuspid, bone maturation.

Fecha de recepción: 15 de octubre de 2012.

Aceptado para publicación: 10 de diciembre de 2012.

Ríos Villasis LK, Soldevilla Galarza L. Relación entre los estadios de maduración esqueletal y calcificación dentaria. Av. Odontoestomatol 2014; 30 (1): 23-28.

\section{INTRODUCCIÓN}

Determinar y comprender los eventos relacionados con el crecimiento y desarrollo es de singular importancia en ortodoncia, esto debido a que los especialistas en el área, actualmente se inclinan a la armonización de las relaciones intermaxilares, mediante la redirección del crecimiento del complejo craneofacial, a través de aparatos de Ortopedia funcional. Para aprovechar al máximo este tipo de tratamiento conocido como Ortodoncia Interceptiva, es necesario reconocer los periodos de aceleración y crecimiento óseo, para corregir desbalances esqueléticos. Si bien es cierto, no podemos estimular o inhibir el crecimiento craneofacial, pero si detectamos la época donde ocurre el pico de crecimiento puberal se puede utilizar la aparatología adecuada, direccionar o eliminar trabamientos que estén impidiendo que eso ocurra (1).

Las medidas del desarrollo humano pueden ser expresadas por la edad cronológica, mental, de estatura, ósea, dentaria, etc. Sin embargo, el único indicador de desarrollo que está disponible desde el nacimiento a la madurez es la edad esquelética (2). La maduración esquelética es uno de los parámetros más confiables para evaluar el crecimiento y desarrollo de un individuo. Diversos investigadores desarrollaron métodos y técnicas para evaluar o valorar la madurez ósea amparándose en el uso de radiografías tomadas en diversas partes del cuerpo (rodilla, pie, mano y muñeca, cadera, vértebras) (3); Sin embargo, la radiografía de mano y muñeca se prefiere porque muestra una mayor cantidad de huesos en desarrollo, además, requiere de un mínimo de exposición radiológica sin afectar el resto del cuerpo (4).
En nuestro ámbito, diferentes parámetros han sido estudiados para determinar la edad dentaria, con la finalidad de demostrar su nivel de confiabilidad para ubicar al paciente en la curva de crecimiento puberal; existen estudios que sugieren que la formación radicular es un indicador de maduración más confiable que la erupción dentaria (5). El método de Dermirjian (6) ha sido utilizado en Ortodoncia en la evaluación de la edad ósea de los pacientes; no se encontró en la literatura reportes de que se utilice en la estimación del potencial de crecimiento del paciente, lo cual nos motivó a evaluar el mismo y determinar la correlación entre los estadios de calcificación de caninos, primeras y segundas premolares inferiores, y los estadios de maduración ósea de Fishman (curva de crecimiento puberal) (7); por lo que se considera que de existir correlación entre los mismos, se contará con una herramienta para determinar el pico de crecimiento puberal, sin la necesidad de utilizar una radiografía adicional de la mano.

El propósito del estudio fue determinar la relación entre los estadios de maduración ósea carpal y calcificación dentaria, para contar con un método confiable y sencillo, como lo es la radiografía panorámica, al momento de realizar el estudio para el diagnóstico y planificación de tratamiento en la historia clínica ortodóncica.

\section{MATERIAL Y MÉTODOS}

Se realizó una investigación de tipo observacional, transversal y correlacional en una muestra de 72 pacientes de ortodoncia de 9 a 15 años de edad que asistieron a la clínica de postgrado de la Universidad 
Nacional Mayor de San Marcos. Se revisó cada historia clínica, se seleccionaron de acuerdo a los criterios de inclusión. Una vez realizado esto, se remitió al paciente al área de Imagenología, previa información y autorización del mismo, con una hoja de interconsulta donde figuró la toma de radiografía panorámica y radiografía Carpal de la mano izquierda.

Dichas radiografías se colocaron por cada paciente en su respectiva historia clínica, adecuadamente rotuladas, indicando fecha de toma de radiografía; así como el nombre y edad del paciente.

La lectura radiográfica se realizó en una habitación oscura y se utilizó un negatoscopio de luz fría, el cual fue cubierto con cartulina negra en los bordes, dejando un espacio para la radiografía. La lectura estuvo a cargo del investigador principal, bajo el asesoramiento de un especialista en el área de radiología, para una adecuada lectura de los estadios analizados. Se confeccionó un manual del trabajo de investigación para la asesora en radiología; a fin de dar conocimiento y homogeneizar los criterios para el análisis de los métodos de Fishman y Demirjian. Se examinaron las radiografías (carpal y panorámica) pertenecientes al mismo paciente, según los estadios de maduración de Fishman (11 estadios) y los estadios de calcificación dentaria según Demirjian (5 estadios), respectivamente. Dicho análisis se realizó, a razón de 10 pares de radiografías (carpal y panorámica) por día durante seis días consecutivos y 12 pares de radiografías el séptimo día.

Se confeccionó una ficha de recolección de datos para el registro de la información utilizada durante la fase de ejecución donde se incluyó: Datos del paciente (nombre, edad cronológica, fecha de toma de radiografías, sexo); gráfico esquemático de la mano y muñeca, de acuerdo al método de Fishman, y tabla donde se anotó el estadio de maduración ósea en la cual se encuentra el paciente; gráfico esquemático de los estadios de calcificación dentaria, según el método de Demirjian, y tabla donde se anotó el estadio de calcificación en la cual se encuentra el paciente para canino, primera y segunda premolar de lado derecho e izquierdo, respectivamente.

El procesamiento de los datos se realizó mediante la utilización de una Laptop Sony Vaio VGN-CS170F procesador Core 2 Duo con sistema operativo Windows 7 Ultimate. Todos los análisis estadísticos fueron realizados con el paquete estadístico SPSS versión 15 y Microsoft Office Excel 2007. Se elaboraron tablas de distribución de frecuencia simple y de contingencia para determinar porcentajes para cada estadio de maduración esquelética, intervalos de edad y sexo. Se utilizó la prueba estadística Coeficiente lineal de Spearman para determinar la correlación entre los Estadios de Maduración ósea Carpal y estadios de Calcificación Dentaria, para cada sexo. Todas las pruebas fueron realizadas con un nivel de significancia del $5 \%$.

\section{RESULTADOS Y DISCUSIÓN}

Se evaluó un total de 144 radiografías (panorámicas y carpales) de los pacientes entre 9 y 15 años que fueron atendidos en la Clínica de Postgrado de Ortodoncia. Se eligieron un total de 72 pares de radiografías que cumplieron con los criterios de inclusión y exclusión, el $56,94 \%(\mathrm{f}=41)$ pertenecieron al sexo masculino, y el $43,06 \%(f=31)$ al sexo femenino.

El presente estudio demuestra que existe una correlación significativa entre los estadios de maduración ósea de Fishman y los estadios de calcificación dentaria de Demirjian para caninos, primeros y segundos premolares inferiores. Los resultados muestran que la correlación más alta respecto a Fishman se dio con el canino inferior izquierdo $(r=0,766)$ y la más baja con el segundo premolar inferior izquierdo $(r=0,581)$, la más alta correlación en cuanto a estadios de calcificación dentaria se dio entre caninos inferiores de ambos lados $(r=0,985)$, como se puede observar en la tabla 1. Estudios similares al de Valverde (8) en niños peruanos quien encuentra la correlación más alta en la segunda premolar inferior derecha, la más baja en el canino inferior derecho y la más alta correlación entre estadios de calcificación dentaria para la segundas premolares mandibulares derechas; las correlaciones halladas en este estudio son mayores a las halladas en el presente estudio. Chertkow y Fatty (9) hallan significativa correlación entre el estadio $G$ de calcificación para el canino mandibular y la calcificación del sesamoideo, en su estudio de 140 sujetos caucásicos. En otro estudio Chertkow (10) esta vez en sujetos negros y 


\begin{tabular}{|c|c|c|c|c|c|c|c|}
\hline \multicolumn{8}{|c|}{$\begin{array}{c}\text { TABLA 1.- CORRELACIÓN ENTRE LOS ESTADIOS DE FISHMAN Y LOS ESTADIOS DE } \\
\text { CALCIFICACIÓN DENTARIA SEGÚN DEMIRJIAN PARA CANINOS, PRIMEROS Y SEGUNDOS } \\
\text { PREMOLARES MANDIBULARES }\end{array}$} \\
\hline & Fishman & Can. der. & Can. izq. & $1^{\text {a }}$ PM der. & $1^{\text {a }} P M$ izq. & $2^{\mathrm{a}} \mathrm{PM}$ der. & $2^{\mathrm{a}} \mathrm{PM}$ izq. \\
\hline Fishman & 1 & $0,755^{* *}$ & $0,766 * *$ & $0,702 * *$ & $0,702 * *$ & $0,617 * *$ & $0,581 * *$ \\
\hline Can. der. & $0,755 * *$ & 1 & $0,985 * *$ & $0,881 * *$ & $0,881 * *$ & $0,739 * *$ & $0,775 * *$ \\
\hline Can. izq. & $0,766^{* * *}$ & $0,985 * *$ & 1 & $0,890 * *$ & $0,890 * *$ & $0,767 * *$ & $0,791 * *$ \\
\hline $1^{\text {a }} \mathrm{PM}$ der. & $0,702 * *$ & $0,881 * *$ & $0,890 * *$ & 1 & $0,974 * *$ & $0,778 * *$ & $0,821 * *$ \\
\hline $1^{\text {a }} P M$ izq. & $0,702 * *$ & $0,881 * *$ & $0,890 * *$ & $0,974 * *$ & 1 & $0,778 * *$ & $0,821 * *$ \\
\hline $2^{\mathrm{a}} \mathrm{PM}$ der. & $0,617 * *$ & $0,739 * *$ & $0,767 * *$ & $0,778 * *$ & $0,778 * *$ & 1 & $0,947 * *$ \\
\hline $2^{a}$ PM izq. & $0,581 * *$ & $0,775 * *$ & $0,791 * *$ & $0,821 * *$ & $0,821 * *$ & $0,947 * *$ & 1 \\
\hline
\end{tabular}

blancos, encuentra que el estadio G para el canino mandibular del lado izquierdo se correlacionaba con el pico de crecimiento puberal en mujeres y hombres de ambas razas. Engstrom (11) en su estudio concluye que la relación entre las etapas de maduración del canino mandibular se asocia mejor con las etapas de osificación en relación a otros dientes. Sierra (12) concluye que existe correlación significativa entre los estadios de maduración ósea en el atlas elaborado por Greulich y Pyle y los estadios de calcificación dentaria según el método propuesto por Nolla, siendo la correlación más alta frente al canino mandibular. Rai (13) evaluó las etapas de madurez esquelética según Hassal y Farmen y los estadios de calcificación dentaria según Demirjian encontrando que le segundo molar es el diente que mostró mayor correlación. Los resultados del presente trabajo señalan que los estadios de calcificación del canino inferior previos al G, en hombres se relacionan con estadios de maduración esquelética anteriores al estadio 7 de Fishman (pico de crecimiento puberal para sexo masculino). Sin embargo, en mujeres se encuentra una equivalencia entre los estadios ascendentes de la curva de crecimiento (del 1 al 5) y los de calcificación dentaria; asimismo, no se encuentra una alta correlación entre el estadio $G$ y los estadios que indican el pico máximo de crecimiento puberal (estadio 6 y estadio 7 de Fishman, para hombres y mujeres, respectivamente); esto podría deberse al tamaño limitado de la muestra que alteran las ocurrencias para cada caso. Demirjian (14) señala que la iniciación del pico de crecimiento puberal coinci- de con el estadio F, el estadio G coincide con la erupción del canino en la cavidad oral y se da un año antes del pico de crecimiento puberal en hombres y cinco meses antes en mujeres. Ossa (15) evaluó los estadios de calcificación dentaria según el método propuesto por Nolla, y la comparó con la osificación de la falange media del dedo medio de la mano derecha, concluyendo que el canino es un buen indicador de maduración. Krailassiri (16) evaluó el desarrollo de caninos, primeros y segundos premolares, y segundos y terceros molares de acuerdo al sistema de Demirjian y lo comparó con los estadios de Fishman; en su estudio encontró que el segundo premolar demostró la mayor correlación y que el estadio $\mathrm{F}$ del canino para ambos sexos coincidió con el $\mathrm{MP}_{3}$. Uysal (17) en su estudio en sujetos de Turquía evaluó las piezas dentarias de la arcada inferior según el método de Demirjian y la maduración esquelética según el método de Bjork; se encontraron correlaciones significativas, el segundo molar mostró la mayor correlación y el tercer molar la más baja; asimismo, la formación de la raíz del canino así como el primer premolar se completo en la mayoría de sujetos en el $M P_{3 \text { cap. }}$

Los resultados, muestran correlación altamente significativa entre el estadio $\mathrm{H}$ de caninos y los estadios de Fishman correspondientes al descenso y final de la curva de crecimiento, para ambos sexos. Coutinho y Buschang (18) hallaron una correlación altamente significativa entre el estadio G de calcificación del canino mandibular izquierdo y el pico de crecimien- 
to puberal según Greulich y Pyle. Los hombres mostraron el canino en estadio G 1,3 años antes del pico de crecimiento puberal y las mujeres 0,4 años. En este estudio la edad promedio cuando se da el pico de crecimiento (estadio 6) para mujeres fue de 11,77 años y 13,95 años (estadio 7). Esto es similar a los encontrados por Fishman en mujeres y hombres (12,06 años y 13,75 años). Para el grupo de 11 años en mujeres tomando como referencia la edad promedio encontrada en este estudio para el pico de crecimiento se observa una mayor ocurrencia del estadio G de Demirjian, como se puede observar en el Gráfico 1; mientras que para el grupo de 13 años en hombres de igual manera tomando en cuenta la edad promedio de 13,95 años se observa una mayor ocurrencia del estadio $\mathrm{H}$ de Demirjian, y una mayor ocurrencia del estadio $\mathrm{G}$ con el estadio 4 de Fishman (calcificación del sesamoideo), como se puede observar en el Gráfico 2. Gupta (19), en su estudio realizado en la India, mostró una correlación signifi-

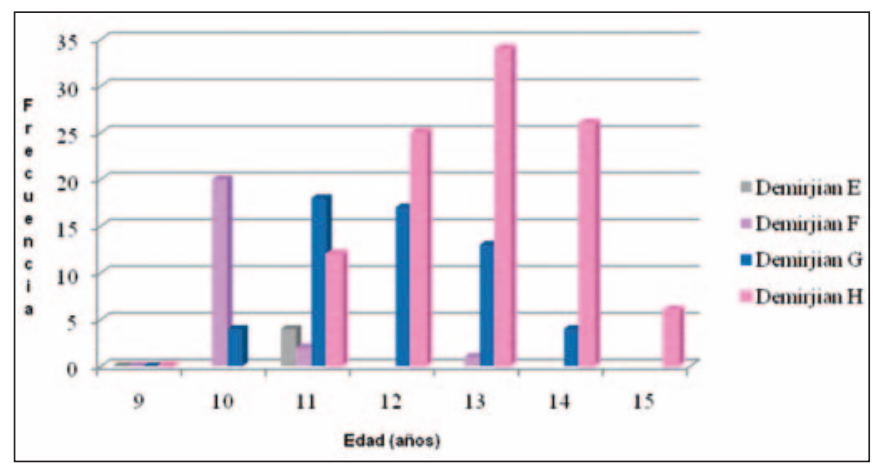

Gráf. 1. Frecuencia en número de los estadios de Demirjian según edad cronológica, para el sexo femenino.

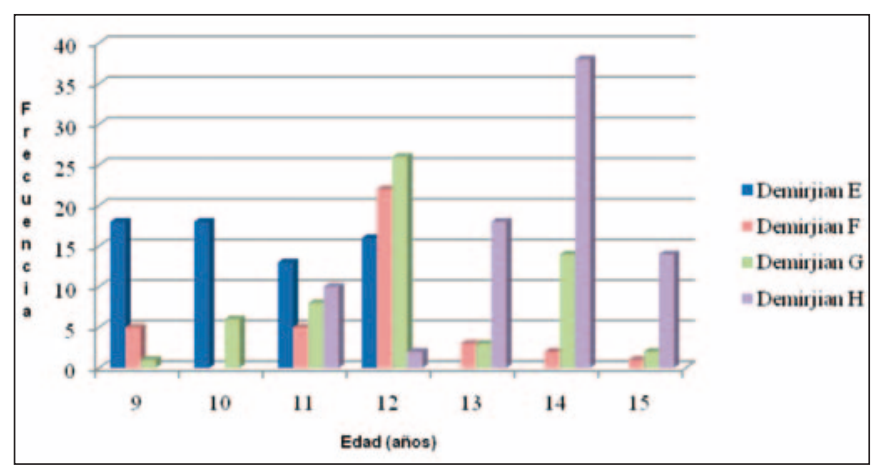

Gráf. 2. Frecuencia en número de los estadios de Demirjian según edad cronológica, para el sexo masculino. cativa entre el estadio de calcificación G en caninos y la aparición del hueso sesamoideo en niñas; sin embargo, estos resultados no son aplicables a niños debido a que al momento de aparición del sesamoideo cubital, el cierre apical ya era manifiesto.

Por consiguiente, la correlación demostrada por los caninos, primeras y segundas premolares inferiores respecto a los estadios de Fishman (curva de crecimiento puberal maxilar y mandibular) fue significativa para ambos sexos. Se encontró correlación significativa $(r=0,766, p<0,05)$ entre los estadios de calcificación de caninos, primeros y segundos premolares inferiores, con los estadios de maduración ósea de Fishman.

\section{BIBLIOGRAFÍA}

1. Meredith HV. Research studies on growth of the body and Face. Am J Orthod 1959;45:110-24.

2. Gilli G. The assessment of skeletal maturation. Horm Res 1996;45(2):49-52.

3. Kucukkeles N, Acar A, Biren S, Arun T. Comparisons between cervical vertebrae and hand-wrist maturation for the assessment of skeletal maturity. J Clin Pediatr Dent 1999;24(1): 47-52.

4. Gibilisco JA. Diagnóstico radiológico en estomatología. $5^{a}$ edición. Editorial Panamericana. Buenos Aires, 1988.

5. Coutinho S. Miranda F. Relation ships between mandibular canine calcification stages ande skeletal maturity. Am J Orthod Dentofac Othop 1994;114(4):429-7.

6. Demirjian A, Buschang Ph, Tanguay R, Kingnorth D. Interrelationships among measures of somatic, skeletal, dental, and sexual maturity. Am J Orthod Dentofacial Orthop 1985;88:433-8.

7. Mayari G, Otaño R. Concordancia entre los estadios de maduración esquelética y los estadios de calcificación dental. Revista Cubana de Estomatología 2010;47(2)207-14. 
8. Valverde R, Adriazola M, Meneses A. Correlación entre estadios de calcificación de caninos y segundas premolares mandibulares con la curva de crecimiento puberal maxilar y mandibular. Rev. Estomatol Herediana 2004;14(1-2):12-17.

9. Chertkow S, Fatti P. The relationship between tooth mineralization and early radiographic evidence of the ulnar sesamoid. Angle Orthod 1979;49(4): 282-8.

10. Chertkow S. Tooth mineralization as an indicator of the puberal growth spurt. Am J Orthod 1980; 77(1):79-91.

11. Malave Y, Rojas I, Análisis carpal como indicador de maduración ósea. Acta Odontológica Venezolana 2000;38(3):4-9.

12. Sierra AM. Assessment of dental and skeletal maturity. A new approach. Angle Orthod 1987;57 (3):194-208.

13. Rai B. Relationship of Dental and skeletal Radiograph: Maturity Indicator. The Internet Journal of Biological Anthropology 2008; 2(1). DOI: 10.5580/1aa0. Disponible en: http://archive. ispub.com/journal/the-internet-journal-ofbiological-anthropology/volume-2-number-1/ relationship-of-dental-and-skeletal-radiographmaturity-indicator.html\#sthash.L49hStcM.dpbs.

14. Demirjian A, Buschang Ph, Tanguay R, Kingnorth D. Interrelationships among measures of somatic, skeletal, dental, and sexual maturity. Am J Orthod Dentofacial Orthop.1985;88:433-8.

15. Ossa Ja, Puerta A, Cortés N. Calcificación dentaria como indicador del crecimiento prepuberal. Rev. Fac. Odont. Univ. Ant 1996;8(1):16-21.

16. Uysal T, Sari Z, Ramoglu Si, Basciftci FA. Relationships between dental and skeletal maturity in Turkish subjects. Angle Orthod 2004 Oct;74(5):657-64.

17. Krailassiri S, Anuwongnukroh N, Dechkunakorn S. Relationships between dental calcification stages and skeletal maturity indicators in Thai individuals. Angle Orthod 2002 Apr;72(2):155-66.

18. Continho S. Bushgang P. Relationships between mandibular canine calcification stages and skeletal maturity. Am J Orthod Dentofac Orthop 1993;104(3):262-8.

19. Gupta S. Assessment of puberty growth spurt in boys and girls: a dental radiographic method. J Indian Soc Pedod Prev Dent 1995;13(1):4-9.

\section{CORRESPONDENCIA}

Liz Katty Ríos Villasis

Jr. Mogaburos, 112. Jesús María.

San Marcos

Correo electrónico: kattyriosv@hotmail.com 